\title{
EFEITO DE PÓS VEGETAIS SOBRE SITOPHILUS ZEAMAIS (MOTS., 1855) (COLEOPTERA: CURCULIONIDAE)
}

\author{
A. Lima-Mendonça ${ }^{1}$, S.M.F. Broglio ${ }^{2}$, A.M.N. de Araújo ${ }^{3}$, D.O.P. Lopes ${ }^{4}$, N.S. Dias-Pini ${ }^{5}$ \\ ${ }^{1}$ Universidade Federal de Alagoas, Rede Nordeste de Biotecnologia, Av. Lourival Melo Mota, s/no, CEP \\ 57072-900, Maceió, AL, Brasil. E-mail: lanazte@yahoo.com.br
}

\section{RESUMO}

\begin{abstract}
Objetivou-se testar a atividade inseticida de pós vegetais em Sitophilus zeamais. Foram testados os pós de Anadenanthera colubrina (folhas); Annona muricata (sementes); Azadirachta inidica (folhas e flores); Caesalpinia pyramidalis (folhas), Chenopodium ambrosioides (folhas e flores); Cymbopogon sp. (folhas); Cymbopogon citratus (folhas); Momordica charantia (folhas e frutos); Piper nigrum (sementes); e Ricinus communis (folhas). Além disso, avaliou-se o potencial inseticida de folhas e flores de C. ambrosioides em diferentes dosagens. Na avaliação de repelência foi estabelecido um índice de preferência, e utilizado o teste t para comparação das médias das espécies vegetais. Também foi realizada a comparação das médias das plantas que foram classificadas como repelentes. Para avaliação da mortalidade, procedeu-se a análise de variância e a comparação das médias pelo teste de Tukey e também o teste $t$ para comparação das médias dos tratamentos C. ambrosioides e P. nigrum. Os dados de emergência foram analisados pelo teste de Tukey. Para avaliar os dados de mortalidade, ocasionados por $C$. ambrosioides, determinou-se a $\mathrm{CL}_{50}$ utilizando a análise de Probit. Os dados de emergência foram verificados pela análise de regressão. As plantas que provocaram repelência foram Cymbopogon sp., C. citratus e C. ambrosioides. A planta que mais afetou a sobrevivência da praga foi C. ambrosioides, que provocou mortalidade total dos insetos infestantes e nenhuma emergência. Adultos de $S$. zeamais são mais suscetíveis a concentração de $0,125 \mathrm{~g}$ do pó de C. ambrosioides.
\end{abstract}

PALAVRAS-CHAVE: Inseticidas de origem vegetal, toxidez, gorgulho do milho.

\begin{abstract}
EFFECT OF PLANT POWDERS ON SITOPHILUS ZEAMAIS (MOTS., 1855) (COLEOPTERA: CURCULIONIDAE). The objective of the present study was to test the insecticidal activity of vegetable powders on Sitophilus zeamais. Powders of Anadenanthera colubrina (leaves); Annona muricata (seed); Azadirachta inidica (leaves and flowers); Caesalpinia pyramidalis (leaves); Chenopodium ambrosioides (leaves and flowers); Cymbopogon sp. (leaves); Cymbopogon citratus (leaves); Momordica charantia (leaves and fruits); Piper nigrum (seed); and Ricinus communis (leaves) were evaluated. In addition, we evaluated the insecticidal potential of leaves and flowers of C. ambrosioides at different dosages. In the evaluation of repellency a preference index was established, and the $t$ test was used to compare the means of plant species. The means of plants that were classified as repellent were also compared. To assess mortality, we proceeded with the analysis of variance and comparison of means by Tukey test and also the $t$ test for comparing the means of the C. ambrosioides and $P$. nigrum treatments. The emergence data were analyzed by Tukey test. To evaluate the data on mortality caused by $C$. ambrosioides, the $\mathrm{CL}_{50}$ was determined using Probit analysis. The emergence data were verified by regression analysis. Plants that caused repellency were Cymbopogon sp., C. citratus and C. ambrosioides. The plant that most affected the survival of the pest was C. ambrosioides, which caused total mortality of insect infestations and no adult emergence. Adults of $S$. zeamais are most susceptible to the concentration of $0.125 \mathrm{~g}$ of $C$. ambrosioides powder.
\end{abstract}

KEY WORDS: Natural insecticides, toxicity, maize weevil.

${ }^{2}$ Universidade Federal de Alagoas, Centro de Ciências Agrárias, Rio Largo, AL, Brasil.

${ }^{3}$ Universidade Federal Rural de Pernambuco, Departamento de Agronomia, Recife, PE, Brasil.

${ }^{4}$ Universidade Estadual Paulista, Departamento de Entomologia, Jaboticabal, SP, Brasil.

${ }^{5}$ Empresa Brasileira de Pesquisa Agropecuária/CNPAT, Fortaleza, CE, Brasil.

Arq. Inst. Biol., São Paulo, v.80, n.1, p.91-97, jan./mar., 2013 


\section{INTRODUÇÃO}

No Brasil, o milho é a cultura mais amplamente difundida e cultivada, pois se adapta aos mais diferentes ecossistemas. A produção brasileira de grãos de milho para a safra 2010/2011 foi estimada em 56,33 milhões de toneladas (CompanHia..., 2011).

Omilhoéum dos principais produtos no mercado de alimentos e também é utilizado como fécula, óleo, alimentos in natura e alimentos industrializados. Condições impróprias de armazenagem levam a severos ataques de pragas de grãos armazenados tornando-os impróprios para o consumo (MicHELRAJ; SHARMA, 2006).

Dentre as pragas associadas aos grãos armazenados no Brasil, o gorgulho Sitophilus zeamais (Mots.,1855) (Coleoptera: Curculionidae) destacase como uma das mais significativas, devido ao número de hospedeiros, elevado potencial biótico, capacida-de de penetração na massa de grãos e infestação cruzada, ocasionando danos, sobretudo nas sementes e grãos de milho, arroz e trigo (GALLO et al., 2002).

O controle químico desta praga é, geralmente, efetuado com inseticidas fumegantes ou de contato que, apesar de eficazes, podem causar intoxicações aos aplicadores, presença de resíduos tóxicos nos grãos e seleção de populações resistentes (BENHALIMA et al., 2004). A preocupação dos consumidores em relação ao ambiente, à qualidade do alimento e aos efeitos colaterais causados pelos inseticidas sintéticos têm incentivado os pesquisadores a testar alternativas para o controle de pragas de grãos armazenados, como os inseticidas de origem vegetal obtidos de plantas (Moreira et al., 2007; RAJENDRAN; SRIRANJINI 2008). Esses produtos geralmente são de baixa toxicidade para o homem e animais, têm menor custo, degradam-se rapidamente no ambiente e não contaminam os alimentos com resíduos tóxicos (IsMAN, 2006).

O Brasil abriga uma grande diversidade de plantas de várias famílias botânicas, como Cheno- podiaceae, Verbenaceae, Compositae, Piperaceae, Asteraceae, Lauraceae, Myrtaceae, entre outras, que possuem compostos secundários bioativos com propriedades inseticidas (TAPONDJOU et al., 2002, EsTRELA et al. 2006; RAJENDRAN; SRIRANJINI 2008).

Considerando-se a importância econômica de $S$. zeamais como inseto-praga do milho armazenado e visando diminuir os efeitos indesejáveis do uso de inseticidas sintéticos, foi desenvolvido este trabalho com objetivo de avaliar o efeito do pó de espécies vegetais na repelência, sobrevivência e emergência de adultos de $S$. zeamais, bem como avaliar o efeito do pó de C. ambrosioides L. em diferentes doses.

\section{MATERIAL E MÉTODOS}

Os ensaios foram conduzidos no Laboratório de Entomologia, do Centro de Ciências Agrárias da Universidade Federal de Alagoas, à temperatura média de $28,5^{\circ} \mathrm{C} \pm 2^{\circ} \mathrm{C}$, umidade relativa de $79,2 \%$ $\pm 10 \%$ e fotofase de $12 \mathrm{~h}$.

Os insetos utilizados nos ensaios foram provenientes da criação mantida em laboratório, em grãos de milho, utilizando-se recipientes plásticos $(9,0$ $\mathrm{cm}$ de diâmetro $\times 7,5 \mathrm{~cm}$ de altura) fechados com tampas perfuradas.

Foram coletas partes vegetais novas, de espécies vegetais na idade adulta (Tabela 1 ). As plantas foram identificadas no herbário MAC do Institudo do Meio Ambiente. O material vegetal foi transferido para estufa para secagem a $40^{\circ} \mathrm{C}$, sendo em seguida passado em moinho de facas obtendo-se um pó fino.

A coleta foi realizada em quatro municípios do Estado de Alagoas: Rio Largo, União dos Palmares, Maceió e São João da Tapera. Os solos dos municípios de Rio Largo e União dos palmares são classificados como Argiloso Vermelho-Amarelo, na Cidade de Maceió Latossolo Vermelho-Amarelo, e Neossolo Regolítico é o solo predominante em São José da Tapera.

Tabela 1 - Espécies, nomes comuns e partes utilizadas das plantas testadas para Sitophilus zeamais.

\begin{tabular}{lcc}
\hline Espécie vegetal & Nome comum & Partes utilizadas \\
\hline Anadenanthera colubrina Vell. & Angico & Folhas \\
Annona muricata L. & Graviola & Sementes \\
Azadirachta indica A. Juss. & Nim & Folhas e flores \\
Caesalpinia pyramidalis Tul. & Catingueira & Folhas \\
Chenopodium ambrosioides L & Mastruz & Folhas e flores \\
Cymbopogon sp. & Citronela & Folhas \\
Cymbopogon citratus Stapf. & Capim-Santo & Folhas \\
Momordica charantia L. & Melão-de-São-Caetano & Folhas e frutos \\
Piper nigrum L. & Pimenta-do-reino & Sementes \\
Ricinus communis L. & Mamona & Folhas \\
\hline
\end{tabular}




\section{Avaliação da repelência sobre adultos de $S$. zeamais}

O delineamento experimental foi inteiramente casualizado com 10 repetições. Cada espécie vegetal foi testada isoladamente, utilizando-se uma arena contendo 5 caixas plásticas circulares $(7,0 \mathrm{~cm}$ de diâmetro $\times 4,5 \mathrm{~cm}$ de altura), sendo uma central interligada às demais por cilindros plásticos (Fig. 1). Em duas das caixas foram colocados $10 \mathrm{~g}$ de milho, misturados com $1,0 \mathrm{~g}$ de pó da espécie vegetal em teste. Na caixa central foram liberados 20 adultos de $S$. zeamais não-sexados. Nas demais caixas (testemunhas), foram colocados apenas o substrato alimentar (grãos de milho). Após 24h, foi contado o número de insetos por recipiente.

Para comparação dos tratamentos, foi estabelecido um Índice de Preferência (I.P.), em que: I.P. $=(\%$ de insetos na planta-teste $-\%$ de insetos na testemunha) $/(\%$ de insetos na planta-teste $+\%$ de insetos na testemunha), no qual: I.P.: $-1,00$ a $-0,10$, planta-teste repelente; I.P.: $-0,10$ a $+0,10$, plantateste neutra; I.P.: $+0,10$ a $+1,00$, planta-teste atraente (Procópio et al., 2003).

Os dados foram analisados utilizando-se o teste Shapiro-Wilk para homogeneidade de variância, apresentando normalidade. Foi utilizado o teste $\mathrm{t}$ ao nível de $5 \%$ de probabilidade, para comparação de médias dos tratamentos e suas respectivas testemunhas, através do Programa Sisvar versão 5.1 (FERREIRA, 2007). Foi realizada também a comparação das médias das espécies vegetais que foram classificadas como repelente, utilizando o Programa SAS versão 9.0 (SAs Institute, 2002).

\section{Avaliação da atividade inseticida}

Para avaliar o efeito dos pós vegetais na sobrevivência e emergência, foram utilizados recipientes plásticos $(7,0 \mathrm{~cm}$ de diâmetro e $4,5 \mathrm{~cm}$ de altura), contendo cada um deles $10 \mathrm{~g}$ de grãos de milho e $1,0 \mathrm{~g}$ do pó da espécie vegetal em teste. Em cada recipiente foram colocados 20 adultos não-sexados com idade de até cinco dias. Cada bioensaio foi conduzido no delineamento inteiramente casualizado, constituído de 10 repetições.

A sobrevivência dos adultos foi avaliada diariamente, até o oitavo dia após a instalação do experimento. Na avaliação de emergência, decorridos 5 dias de confinamento, os insetos foram retirados. Os adultos emergidos foram quantificados e descartados em avaliações diárias, a partir do $34^{\circ}$ dia do confinamento até o término da emergência (cinco dias consecutivos sem emergência).

$\mathrm{Na}$ análise de mortalidade, os tratamentos $C$. ambrosioides (mastruz) eP.nigrum (pimenta-do-reino) foram retirados objetivando-se reduzir a discrepância apresentada devido à alta mortalidade apresentada pelos tratamentos excluídos com relação aos demais. Segundo Oliveira; Vendramim (1999), tal procedimento é comum em entomologia, visando-se homogeneizar os resultados, adequando-os às análises.

Deste modo, os dados foram analisados utilizando-se o teste Shapiro-Wilk para homogeneidade de variância e, consequentemente, transformados em raiz quadrada de $x+0,5$. Em seguida, procedeu-se à análise de variância e à comparação das médias pelo teste de Tukey, ao nível de $5 \%$ de probabilidade pelo Programa SAS versão 9.0 (SAS INSTITUTE, 2002). Foi utilizado o teste t através do Programa Sisvar versão 5.1 (FERREIRA, 2007) para comparação das médias dos tratamentos excluídos desta análise.

Os dados de emergência foram analisados utilizando-se o teste Shapiro-Wilk, apresentando normalidade. Em seguida, foram submetidos à análise de variância e os tratamentos comparados pelo teste de Tukey ao nível de $5 \%$ de probabilidade também utilizando o Programa SAS versão 9.0 (SAS INSTITUTE, 2002).

Efeito do pó de C. ambrosioides na sobrevivência dos adultos de $S$. zeamais

Para C. ambrosioides, foi determinada a atividade inseticida em dosagens progressivamente menores $(0,5 \mathrm{~g} ; 0,25 \mathrm{~g} ; 0,125 \mathrm{~g} ; 0,0625 \mathrm{~g}$ e $0,03125 \mathrm{~g}$ por recipiente contendo $10 \mathrm{~g}$ de grãos de milho), a fim de se determinar a dosagem mínima para controle dos adultos de $S$. zeamais. Utilizou-se uma testemunha contendo apenas o substrato alimentar. Em cada recipiente plástico $(7,0 \mathrm{~cm}$ de diâmetro e $4,5 \mathrm{~cm}$ de altura) foram colocados 20 adultos não-sexados com idade de até 5 dias.

A sobrevivência dos insetos foi avaliada diariamente num período de 5 dias, contando-se e retirando-se os indivíduos mortos. Esse experimento foi instalado no delineamento experimental inteiramente casualizado, sendo utilizadas 10 repetições para os tratamentos e testemunha.

Os dados foram submetidos à análise de Probit, utilizando o Programa SAS versão 9.0 (SAS INSTITUTE, 2002). Na foi necessário realizar a correção de moralidade, pois não foi observado mortalidade no tratamento testemunha. Foi estimada a $\mathrm{CL}_{50}$ das concentrações na detecção de toxicidade a Sitophilus zeamais.

Efeito do pó de Chenopodium ambrosioides na emergência dos adultos de $S$. zeamais

Para avaliar o efeito do pó de C. ambrosioides na emergência, foram utilizados recipientes plásticos (7,0 cm de diâmetro x 4,5 cm de altura), contendo em cada um deles $10 \mathrm{~g}$ de grãos de milho e dosagens 
progressivamente menores $(0,5 \mathrm{~g} ; 0,25 \mathrm{~g} ; 0,125 \mathrm{~g}$; $0,0625 \mathrm{~g}$ e 0,03125 g) da espécie vegetal em teste, exceto a testemunha, em que foi mantido apenas o substrato alimentar.

Em cada recipiente foram colocados 20 adultos não-sexados com idade de até 5 dias. Decorridos 5 dias de confinamento os insetos foram retirados. Os adultos emergidos foram quantificados e descartados em avaliações diárias, a partir do $34^{\circ}$ dia do confinamento até o término da emergência ( 5 dias consecutivos sem emergência).

Esse bioensaio foi instalado no delineamento experimental inteiramente casualizado, sendo utilizadas dez repetições para cada tratamento e testemunha. Os dados foram avaliados pela análise de regressão (ANAREG) pelo ProgramaSAS versão 9.0 (SAS InSTITUTE, 2002)

\section{RESULTADOS E DISCUSSÃO}

Avaliação da repelência sobre adultos de $S$. zeamais

Foi constatado efeito repelente dos seguintes pós de origem vegetal: Cymbopogon sp. (IP $=-0,55$ ), C. citratus ( $\mathrm{IP}=-0,3$ ) e C. ambrosioides ( $\mathrm{IP}=-0,5)$, já que para esses tratamentos os valores de I.P. foram inferiores a -0,10 (limite estipulado para a plantateste ser considerada repelente), além disso, o teste $t(P>0,05)$ indicou diferença significativa entre os tratamentos e as testemunhas. Nos tratamentos com pó de $C$. pyramidalis e $M$. charantia, em que ambas apresentaram IP $=0,1$, pôde-se constatar efeito atrativo destes pós, pois os valores de IP foram superiores a $+0,10$, não havendo diferença significativa entre os tratamentos e testemunha, de acordo com o teste $\mathrm{t}$ (Tabela 2).

As demais espécies vegetais testadas, cujos índices de repelência estiveram dentro do intervalo de $-0,10$ a $+0,10$, foram consideradas neutras, isto é, sem nenhuma atividade sobre o comportamento de S. zeamais. De acordo com o teste de Tukey, não houve diferença significativa entre as espécies vegetais que apresentaram efeito repelente nos adultos de $S$. zeamais (Tabela 3 ).

TAVARES; VENDRAMIN (2005) não encontrou efeito repelente nem atrativo dos pós das folhas e frutos de C. ambrosioides em relação aos adultos de S. zeamais, enquanto LAGUNES; RodRIGUEZ (1989) observaram efeito repelente dessa planta sobre $S$. zeamais. No que se refere a Cymbopogon sp., o efeito repelente é atribuído à presença de substâncias voláteis em suas folhas, como citronelal, eugenol, geraniol, entre outras, denominadas de um modo geral como monoterpenos (SHASANY et al., 2000).

Tabela 2 - Comparação do efeito repelente entre diferentes pós de origem vegetal em adultos de S. zeamais.

\begin{tabular}{|c|c|c|c|}
\hline Tratamento & Adultos atraídos $(\%)^{1}$ & $\mathrm{IP}^{2}(\mathrm{M} \pm \mathrm{EP})$ & Classificação $^{3}$ \\
\hline A. colubrina & $52,0 \mathrm{a}$ & & \\
\hline Testemunha & $48,0 \mathrm{a}$ & $0,04 \pm 0,60$ & $\mathrm{~N}$ \\
\hline C. citratus & $35,0 \mathrm{~b}$ & & \\
\hline Testemunha & $65,0 \mathrm{a}$ & $-0,3 \pm 0,68$ & $\mathrm{R}$ \\
\hline C. pyramidalis & $55,0 \mathrm{a}$ & & \\
\hline Testemunha & $45,0 \mathrm{a}$ & $0,1 \pm 0,87$ & A \\
\hline Cymbopogon sp. & $22,5 b$ & & \\
\hline Testemunha & $77,5 \mathrm{a}$ & $-0,55 \pm 0,93$ & $\mathrm{R}$ \\
\hline A. muricata & $43,5 a$ & & \\
\hline Testemunha & $56,5 \mathrm{a}$ & $-0,13 \pm 0,93$ & $\mathrm{~N}$ \\
\hline R. communis & $52,0 \mathrm{a}$ & & \\
\hline Testemunha & $48,0 \mathrm{a}$ & $0,04 \pm 0,96$ & $\mathrm{~N}$ \\
\hline C. ambrosioides & $25,0 \mathrm{~b}$ & & \\
\hline Testemunha & $75,0 \mathrm{a}$ & $-0,5 \pm 0,75$ & $\mathrm{R}$ \\
\hline M. charantia & $55,0 \mathrm{a}$ & & \\
\hline Testemunha & $45,0 \mathrm{a}$ & $0,1 \pm 0,93$ & $\mathrm{~A}$ \\
\hline A. inidica & $48,5 \mathrm{a}$ & & \\
\hline Testemunha & $51,5 a$ & $-0,03 \pm 0,85$ & $\mathrm{~N}$ \\
\hline P. nigrum & $38,5 a$ & & \\
\hline Testemunha & $61,5 a$ & $-0,23 \pm 1,14$ & $\mathrm{~N}$ \\
\hline
\end{tabular}


Tabela 3 - Comparação do efeito repelente entre diferentes pós de origem vegetal em adultos de S. zeamais.

\begin{tabular}{lr}
\hline Tratamento & Adultos repelidos $(\%)^{1}$ \\
\hline Cymbopogon sp. (citronela) & $77,50 \pm 0.93 \mathrm{a}$ \\
C. ambrosioides (mastruz) & $75,00 \pm 0,76 \mathrm{ab}$ \\
C. citratus (capim-santo) & $65,00 \pm 0,68 \mathrm{abc}$ \\
\hline
\end{tabular}

${ }^{1}$ Dados médios provenientes de cinco repetições no delineamento inteiramente casualizado, é dias $( \pm \mathrm{EP})$ seguidas da mesma letra não diferem entre si pelo teste de Tukey $(\mathrm{P}=0,05)$.

Efeito dos pós das espécies vegetais na sobrevivência e emergência dos adultos de $S$. zeamais

O pó de C. ambrosioides foi o único que ocasionou mortalidade de $100 \%$ dos adultos de S. zeamais no primeiro dia após a infestação e diferiu estatisticamente do pó da P. nigrum L. que ocasionou a morte de $89 \%$ dos adultos após o oitavo dia de contato. Os demais tratamentos apresentaram um efeito intermediário na mortalidade e algumas espécies vegetais não diferiram da testemunha (Tabelas 4 e 5).

MAREDIA et al. (1992) encontraram baixa sobrevivência desse inseto em substrato tratado com óleo de sementes denim. Oefeito tóxico de C. ambrosioides sobre S. zeamais também foi constatado por PrOCóPIO et al. (2003), provocando mortalidade total dos adultos do gorgulho, após dez dias do contato com pós de seis espécies vegetais. No mesmo trabalho foi observado que o pó de $R$. communis não diferiu da testemunha e apresentou mortalidade de $13,33 \%$ dos adultos de $S$. zeamais.

No que se refere à emergência de $S$. zeamais (Tabela 6), verificou-se que o pó de C. ambrosioides foi o único tratamento em que não ocorreu a emergência de adultos, o que pode ser explicado pela total mortalidade dos insetos já no primeiro dia de contato com o pó dessa planta, seguido de Piper nigrum que não diferiu estatisticamente $(P>0,05)$ do pó de $C$. ambrosioides, apresentando um número médio de 1,1 adultos emergidos. Os tratamentos C. citratus e A. colubrina foram considerados intermediários, com média de emergência de 11,2 e 13,5 adultos, respectivamente.

Tabela 4 - Efeito (Média \pm EP) de pós vegetais na mortalidade de $S$. zeamais, após o oitavo dia de contato.

\begin{tabular}{lc}
\hline Tratamentos & Mortalidade $(\%)^{1}$ \\
\hline Chenopodium ambrosioides L. (mastruz) & $100,00 \pm 0,00 \mathrm{a}$ \\
Piper nigrum L. (pimenta-do-reino) & $89,00 \pm 0,41 \mathrm{~b}$ \\
\hline${ }^{1}$ Dados médios provenientes de dez repetições no delin- \\
eamento inteiramente casualizado, Médias $( \pm \mathrm{EP})$ seguidas \\
da mesma letra não diferem entre si pelo teste $\mathrm{t}(\mathrm{P}=0,05)$.
\end{tabular}

Nos demais tratamentos, o número médio de adultos emergidos não diferiu $(P>0,05)$ do valor médio encontrado na testemunha. MAKanjuOla (1989), investigando a ação inseticida de extratos de folhas e sementes de $A$. indica, constatou que todos os extratos reduziram a emergência de adultos de $S$. zeamais e Sitophilus oryzae (LINNAEUs, 1763), diferindo do resultado observado neste trabalho, no qual foi avaliado o pó dessa espécie vegetal. Procópio et al. (2003) verificaram que o pó das folhas, flores e frutos de $C$. ambrosioides foi o único tratamento em que não ocorreu emergência de adultos de S. zeamais, como observado neste trabalho.

Efeito do pó da espécie vegetal Chenopodium ambrosioides na sobrevivência dos adultos de $S$. zeamais

A curva de concentração-mortalidade do pó de Chenopodium ambrosioides apresentou média de 4,393 $\pm 0,241\left(X^{2}=4,26 ; G L=3 ; P=0,2338\right)$ (Tabela 7). O alto valor de inclinação demonstra que adultos de $S$. zeamais responderam de forma homogênea às concentrações aplicadas, ou seja, pequenas variações na concentração do pó promoveram grandes variações na mortalidade.

$\mathrm{O}$ valor da $\mathrm{CL}_{50}$ estimado pela análise de Probit para o pó de C. ambrosioides foi de 0,113 (IC I $_{95} \%$ 0,89$1,22)$ gramas de pó (Tabela 7). Isso demonstrou que os adultos de $S$. zeamais foram mais susceptíveis a dosagem de $0,125 \mathrm{~g}$ do pó.

MAZZONETTo (2002), a partir de testes com concentrações de pó de C. ambrosioides variando de 0,007 a 0,019 para avaliar a sucetibilidade de Zabrotes subfasciatus (Вон., 1833) (Coleoptera: Bruchidae), estimou $\mathrm{CL}_{50}$ de $0,018 \mathrm{~g}$ do pó. Na comparação dos valores das $\mathrm{CL}_{\mathrm{s} 50}$ estimados por MAZZONETTO (2002) e neste trabalho, pode-se constatar a maior eficiência do pó dessa espécie vegetal para Z. subfasciatus do que para S. zeamais.

Tabela 5 - Efeito (Média \pm EP) de pós vegetais na mortalidade de $S$. zeamais, após o oitavo dia de contato.

\begin{tabular}{lc}
\hline Tratamentos & Mortalidade $(\%)^{1}$ \\
\hline A. muricata (Graviola) & $14,00 \pm 0,19 \mathrm{c}$ \\
C. pyramidalis (Catingueira) & $12,50 \pm 0,18 \mathrm{c}$ \\
R. communis (Mamona) & $11,50 \pm 0,16 \mathrm{c}$ \\
212Cymbopogon sp. (Citronela) & $7,00 \pm 0,20 \mathrm{~cd}$ \\
M. charantia (Melão-de-São-Caetano) & $4,00 \pm 0,09 \mathrm{~cd}$ \\
A. inidica (Nim) & $4,00 \pm 0,12 \mathrm{~cd}$ \\
C. citratus (Capim santo) & $3,00 \pm 0,13 \mathrm{~cd}$ \\
A. colubrina (Angico) & $2,50 \pm 0,10 \mathrm{~cd}$ \\
\hline Testemunha & $0,00 \pm 0,00 \mathrm{~d}$ \\
\hline${ }^{1}$ Dados médios provenientes de dez repetições no delinea- \\
mento inteiramente casualizado, transormados em raiz $(\mathrm{x}$ \\
+ 0,5). Médias ( \pm EP) seguidas da mesma letra não diferem \\
entre si pelo teste de Tukey (P = 0,05)
\end{tabular}


Procópio (2003) estimou que a concentração mínima do pó de $C$. ambrosioides que provoca $100 \%$ de mortalidade de adultos de S. zeamais é de $0,1645 \mathrm{~g}$, resultado semelhante ao obtido neste trabalho.

Efeito do pó da espécie vegetal Chenopodium ambrosioides na emergência dos adultos de $S$. zeamais

Não se constatou emergência de $S$. zeamais nas dosagens de 0,$125 ; 0,25$ e 0,5 de pó/10 $\mathrm{g}$ de grãos de milho $\left(\mathrm{F}_{3,39}=30,98 ; \mathrm{P}<0,0001\right)$. Com a redução da dosagem a emergência aumentou gradativamente, atingindo em média 2,80 e 10,13 de adultos emergidos, nas dosagens de $0,0625 \mathrm{~g}$ e $0,03125 \mathrm{~g}$, respectivamente; na testemunha houve, em média, emergência de 24 adultos de $S$. zeamais. Também foi observada uma correlação positiva pelo modelo linear entre a emergência e as dosagens utilizadas $\left(y=32,62 x-11,83 ; R^{2}=0,324\right)$ (Fig. 1).

Esse aumento de emergência com a redução da dosagem do pó de C. ambrosioides pode ser devido à diminuição da concentração de ascaridol, monoterpeno responsável pela toxidez dessa espécie vegetal (SousA et al., 1991).
Tabela 6 - Efeito (Média \pm EP) de pós vegetais na emergência de $S$. zeamais.

\begin{tabular}{|c|c|}
\hline Tratamentos & $\begin{array}{c}\text { № de adultos } \\
\text { emergidos }^{1}\end{array}$ \\
\hline M.charantia(Melão-de-São-Caetano) & $18,2 \pm 2,27 \mathrm{ab}$ \\
\hline A. muricata (Graviola) & $17,2 \pm 2,24 \mathrm{ab}$ \\
\hline A. inidica (Nim) & $17,1 \pm 2,32 \mathrm{ab}$ \\
\hline C. pyramidalis (Catingueira) & $16,9 \pm 2,10 \mathrm{ab}$ \\
\hline R. communis (Mamona) & $16,3 \pm 2,06 \mathrm{ab}$ \\
\hline Cymbopogon sp. (Citronela) & $16,0 \pm 2,70 \mathrm{ab}$ \\
\hline A. colubrina (Angico) & $13,5 \pm 1,56 b$ \\
\hline C. citratus (Capim santo) & $11,2 \pm 1,95 b$ \\
\hline P. nigrum (pimenta-do-reino) & $1,1 \pm 0,55 c$ \\
\hline C. ambrosioides (Mastruz) & $0,0 \pm 0,00 c$ \\
\hline Testemunha & $24,0 \pm 2,19 a$ \\
\hline
\end{tabular}

${ }^{1}$ Dados médios provenientes de cinco repetições no delineamento inteiramente casualizado, Médias $( \pm \mathrm{EP})$ seguidas da mesma letra não diferem entre si pelo teste de Tukey $(P=0,05)$.

\section{CONCLUSÕES}

Os pós de Cymbopogon sp. (citronela), C.ambrosioides (mastruz) e C. citratus (capim-santo) provocam efeito repelente nos adultos de $S$. zeamais.

Tabela 7 - Dados de inclinação \pm EP das curvas de concentração-mortalidade $\mathrm{CL}_{50}$, $\mathrm{X}^{2}$, probabilidade dos pós de Chenopodium ambrosioides, após cinco dias de contato, em adultos de Sitophilus zeamais.

\begin{tabular}{lcccccc}
\hline Pó vegetal & $\mathrm{GL}^{1}$ & $\mathrm{n}^{2}$ & Inclinação (média $\pm \mathrm{EP}$ ) & $\mathrm{CL}_{50}\left(\mathrm{IC}_{95 \%}\right)^{3}$ & $\mathrm{X}^{2}$ & $P^{4}$ \\
\hline C. ambrosioides & 3 & 1000 & $4,393 \pm 0,241$ & $0,113(0,106-0,120)$ & 4,26 & 0,2338 \\
\hline
\end{tabular}

EP: Erro-padrão; CL: Concentração letal; X²: Qui-quadrado.

${ }^{1} \mathrm{GL}$ : Graus de liberdade.

${ }^{2} \mathrm{n}$ : Número de insetos utilizados no teste.

${ }^{3}$ IC: Intervalo de confiança.

${ }^{4} \mathrm{P}$ : Probabilidade $>0,05$.

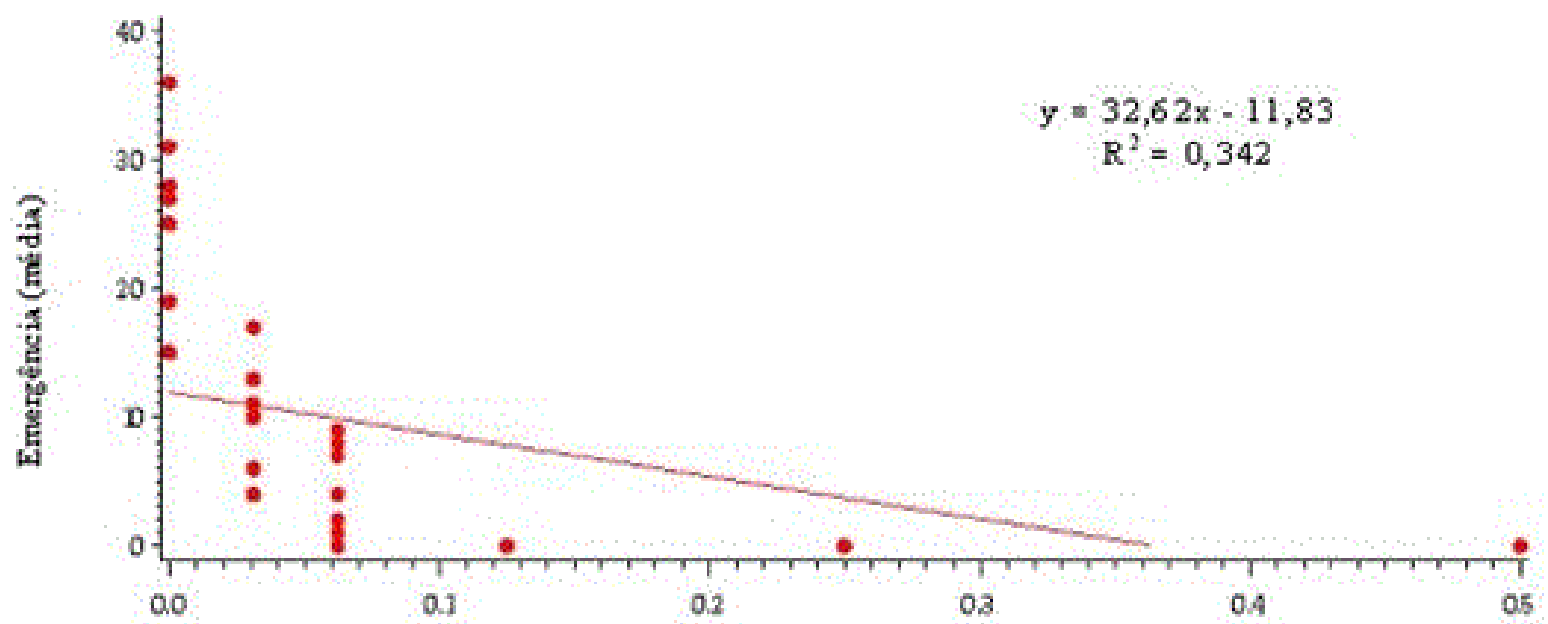

Dose de C. ambrosioides (z pó $/ 20 \mathrm{~g}$ de gräos de milho)

Fig. 1 - Mortalidade de Sitophilus zeamais cinco dias após o contato com grãos de milho tratados com diferentes dosagens de pó de Chenopodium ambrosioides. 
A espécie vegetal mais eficiente, quanto ao efeito inseticida, é Chenopodium ambrosioides L. (mastruz);

Adultos de $S$. zeamais são mais suscetíveis a concentração de $0,125 \mathrm{~g}$ do pó de C. ambrosioides.

\section{AGRADECIMENTOS}

À Fundação de Amparo à Pesquisa do Estado de Alagoas (FAPEAL) pelo apoio financeiro.

\section{REFERÊNCIAS}

BENHALIMA, H.; CHAUDHRY, M.Q.; MILLS, K.A.; PRICE, N.R. Phosphine resistance in stored-product insects collected from various grain storage facilities in Marocco. Journal of Stored Products Research, v.40, p.241-249, 2004.

\section{COMPANHIA NACIONAL DE ABASTECIMENTO} (Brasil). Acompanhamento da safra brasileira. Disponível em: <http://www.conab.gov.br/conabweb/ download/safra/9graos_08.09.pdf>. Acesso em: 29 de ago. 2011.

ESTRELA, J. L. V.; FAZOLIN, M.; CATANI, V.; ALÉCIO, M. R.; LIMA, M. S. Toxicidade de óleos essenciais de Piper aduncum e Piper hispidinervum em Sitophilus zeamais. Pesquisa Agropecuária Brasileira, v.41, n.2, p.217222, 2006.

FERREIRA, D.F. Programa Sisvar versão 5.1. Lavras: Departamento de Ciências Exatas da Universidade Federal de Lavras, 2007.

GALLO, D.; NAKANO, O.; SILVEIRA NETO, S.; CAR-VALHO, R. P. L.; BAPTISTA, G. C.; BERTI FILHO, E.; PARRA, J. R. P.; ZUCCHI, R. A.; ALVES, S. B.; VENDRA-MIN, J. D.; MARCHINI, L. C.; LOPES, J. R. S.; OMOTO, C. Entomologia agrícola. Piracicaba: FEALQ, 2002. 920p.

ISMAN, M.B. Botanical insecticides, deterrents, and repellents in modern agriculture and an increasingly regulated world. Annual Review of Entomology, v.51. p.45-66, 2006.

LAGUNES, T.A.; RODRÍGUEZ, H.C. Busqueda de tecnologia apropriada para el combate de plagas del maiz almacenado en condiciones rústicas. Chapingo: [s.n.], 1989. 150p.

MAKANJUOLA, W.A. Evaluation of extracts of neem (Azadirachta indica A. Juss) for the control of some stored product pests. Journal of Stored Products Research, v.25, p.231-237, 1989.

MAREDIA, K.M.; SEGURA, O.L.; MIHM, J.A. Effects of neem, Azadirachta indica, on six species of maize insect pests. Tropical Pest Management, Basingstoke, v.38, p.190-195, 1992.
MAZZONETTO, F. Efeito de genótipos de feijoeiro e de pós de origem vegetal sobre Zabrotes subfasciatus (Boh.) e Acanthoscelides obtectus (Say) (Col.: Bruchidae). 2002. 134p. Tese (Doutorado em Entomologia) - Universidade de São Paulo, Escola Superior de Agricultura "Luiz de Queiroz", Piracicaba, $1992 .$.

MICHELRAJ, S.; SHARMA, R.K. Fumigant toxicity of neem formulations against Sitophilus oryzae and Rhyzopertha dominica. Journal of Agricultural Technology, v.2, n.1, p.1-16, 2006.

MOREIRA, D.M .; PICANÇO, M.C.; BARBOSA, L.C.A.; GUEDES, R.N.C.; CAMPOS, M.R.; SILVA, G.A.; MARTINS, J.C. Plant compounds insecticide activity against coleoptera pests of stored products. Pesquisa Agropecuária Brasileira, v.42, n.7, p. 909-915, 2007.

OLIVEIRA, J.V.; VENDRAMIM, J.D. Repelência de Óleos Essenciais e Pós Vegetais sobre Adultos de Zabrotes subfasciatus (Boh.) (Coleoptera: Bruchidae) em Sementes de Feijoeiro. Anais da Sociedade Entomológica do Brasil, v.3, p.549-555, 1999.

PROCÓPIO, S.O.; VENDRAMIM, J.D.; RIBEIRO JÚNIOR, J.; SANTOS, J.B. Bioatividade de diversos pós de origem vegetal em relação a Sitophilus zeamais Mots. (Coleoptera: curculionidae). Ciência e Agrotecnologia, v.27, n.6, p.1231-1236, 2003.

RAJENDRAN, S.; SRIRANJINI, V. Plant products as fumigants for stored-product insect control. Journal of Stored Products Research, v.44, p.126-135, 2008.

SAS INSTITUTE. SAS user's guide: statistics, version 9.0. Cary: SAS Institute, 2002.

SHASANY, A.K.; LAU, L.K.; PATRA, N.K.; DAROKAR, M.P.; GARG, A.; KUMAR, S.; KHANUJA, S.P.S. Phenotypic and RAPD diversity among Cymbopogon Winterianus Jowitt accessions in relation to Cymbopogon nardus Rendle. Genetic Resources and Crop Evolution, v.47, n.5, p.553-559, 2000.

SOUSA, M.P.; MATOS, M.E.O.; MATOS, F.J.A.; MACHADO, M.I.L.; CRAVEIRO, A.A. (Ed.). Constituintes químicos ativos de plantas medicinais brasileiras. Fortaleza: Edições UFC, 1991. 416p.

TAPONDJOU, L.A. A.; ADLER, C.; BOUDA, H.; FONTEM, D.A. Efficacy of powder and essential oil from Chenopodium ambrosioides leaves as post-harvest grain protectants against six-stored product beetles. Journal of Stored Products Research, v.38, p.395-402, 2002.

TAVARES, M.A.G.C.; VENDRAMIM, J.D. Bioatividade da Erva-de-Santa-Maria, Chenopodium ambrosioides L., sobre Sitophilus zeamais Mots. (Coleoptera: Curculionidae). Neotropical Entomology, v.34, n.2, p.319-323, 2005.

Recebido em 4/9/11

Aceito em 16/1/13 